\title{
Nanomorphology characterization of sterically stabilized polypyrrole-palladium nanocomposite particles
}

\author{
Hiroaki Takeoka ${ }^{1}$, Nobuyuki Fukui ${ }^{1}$, Shinichi Sakurai ${ }^{2}$, Yoshinobu Nakamura ${ }^{1,3}$ and Syuji Fujii ${ }^{1}$
}

The nanomorphology of sterically stabilized polypyrrole-palladium nanocomposite particles synthesized by aqueous chemical oxidative dispersion polymerization has been extensively characterized by transmission electron microscopy (TEM) and smallangle X-ray scattering (SAXS). High-resolution TEM studies revealed that non-spherical nanocomposite particles were produced with a Heywood diameter of $\sim 30 \mathrm{~nm}$ and also confirmed the existence of palladium nanocrystals with a diameter of $5.4 \mathrm{~nm}$ within the particles. SAXS studies on aqueous dispersions of the nanocomposite particles confirmed good colloidal stability and revealed that the average diameters of the Pd nanoparticles and the nanocomposite particles were 5.0 and $23 \mathrm{~nm}$, respectively, which agreed well with the TEM results. Furthermore, their size distributions were revealed by the SAXS technique. The hydrodynamic diameter of the nanocomposite particles determined by dynamic light scattering was larger than those determined by TEM and SAXS, possibly owing to the presence of a hydrated colloidal stabilizer layer on the particle surfaces. Polymer Journal (2014) 46, 704-709; doi:10.1038/pj.2014.44; published online 28 May 2014

Keywords: conducting polymer; dispersion polymerization; nanocomposite; nanoparticle; small-angle X-ray scattering; transmission electron microscopy

\section{INTRODUCTION}

There has been increasing interest in conducting polymer-noble metal nanocomposites because they provide an exciting system with which to investigate the possibility of designing device functionality ${ }^{1,2}$ and also because they exhibit enhanced sensing and catalytic capabilities. Polypyrrole (PPy)-noble metal nanocomposites are among the most promising nanocomposite materials, and there is increasing interest in their synthesis, characterization and application..$^{3-7}$

Recently, some of the present authors ${ }^{8}$ succeeded in the one-step synthesis of sterically stabilized PPy-palladium (Pd) nanocomposite particles by aqueous chemical oxidative dispersion polymerization using $\mathrm{PdCl}_{2}$ as an oxidant in the presence of a colloidal stabilizer. The synthetic method reported in our previous study has several advantages. For example, the reaction can be conducted in environmentally benign aqueous media rather than in volatile organic solvents. In addition, well-dispersed nanocomposite particles can be produced in a single reaction setup at room temperature. The synthesis of PPy-metal nanocomposites in colloidal form is also an attractive strategy to overcome their poor processability and intractability, which are due to their infusible and insoluble character (probably because of some degree of cross-linking of PPy), and to improve their performance. Pd nanoparticles have been used as catalysts for the hydrogenation of unsaturated olefins ${ }^{9,10}$ and in carbon-carbon coupling reactions, including the SuzukiMiyaura and Heck reactions. ${ }^{11-13}$ Therefore, sterically stabilized PPy-Pd nanocomposite particles consisting of Pd nanoparticles and PPy are expected to function as a catalyst. ${ }^{14,15}$

Considering the wide variety of application areas, it is crucial to understand the synthesis-structure-property relationships of conducting polymer-noble metal nanocomposite particles. Characterization of morphology on the nanometer scale is essential to optimize their properties for specific applications. It is especially important to characterize the nanocomposite nanostructure in the wet state because the nanoparticles could potentially be used in aqueous media as catalysts for carbon-carbon coupling reactions. ${ }^{14,15}$ However, only a limited number of studies on the nanomorphology characterization of conducting polymer-metal nanocomposite particles have been reported, and no studies on the characterization of the nanostructure in the wet state have been published.

Herein, we describe the extensive morphology characterization of PPy-Pd nanocomposite particles. High-resolution transmission electron microscopy (TEM), small-angle X-ray scattering (SAXS) and elemental microanalysis were used to characterize the nanocomposite particles in terms of particle size, distribution, chemical composition, nanomorphology and dispersibility in aqueous media.

${ }^{1}$ Department of Applied Chemistry, Faculty of Engineering, Osaka Institute of Technology, Osaka, Japan; ${ }^{2}$ Graduate School of Science and Technology, Kyoto Institute of Technology, Kyoto, Japan and ${ }^{3}$ Nanomaterials Microdevices Research Center, Osaka Institute of Technology, Osaka, Japan

Correspondence: Professor S Sakurai, Graduate School of Science and Technology, Kyoto Institute of Technology, Matsugasaki, Sakyo-ku, Kyoto 606-8585, Japan or Dr S Fujii, Department of Applied Chemistry, Faculty of Engineering, Osaka Institute of Technology, 5-16-1 Omiya, Osaka 535-8585, Japan.

E-mail: shin@kit.ac.jp or s.fujii@chem.oit.ac.jp

Received 15 January 2014; revised 7 April 2014; accepted 8 April 2014; published online 28 May 2014 
Small-angle scattering of electromagnetic waves is a unique tool for non-destructive material testing that provides a means to quantitatively characterize the structural properties of nanoparticles, such as size and distribution. Compared with microscopic observation techniques, the small-angle scattering technique is more reliable in that it produces results that are averaged over a macroscopic sample volume irradiated by the beam of the electromagnetic wave. This method is particularly powerful for quantitative monitoring of the temporal growth of nanoparticles; this non-destructive technique is the only method that provides the opportunity to analyze the timeevolution of structures or characterize dynamic aspects of nanoparticles. SAXS has previously been applied as a tool to evaluate the diameter and size distribution of nanoparticles to monitor the temporal growth of metal nanoparticles, ${ }^{16}$ such as gold, ${ }^{17-19}$ silver, ${ }^{20}$ palladium $^{21,22}$ and rhodium. ${ }^{21}$ Recently, SAXS was also used to characterize the morphologies of vinyl polymer-silica core-shell nanocomposite particles. ${ }^{23,24}$ In these studies, the size distribution was determined by assuming Gaussian ${ }^{17,18,22}$ or Schulz-Zimm distribution functions ${ }^{19,20}$ to analyze the SAXS results (particle scattering). Direct evaluation of the size distribution, which can be determined by TEM, ${ }^{20,21}$ has not yet been performed using SAXS. However, the SAXS technique can be applied to wet samples (for example, solutions and dispersions) to characterize their structure in the wet state, which cannot be accomplished using electron microscopy. In this paper, we will present results from the first trial of the direct evaluation of size distribution, without assuming any mathematical functions, for conducting polymer-noble metal nanocomposite particles and metal nanoparticles in the wet state, based on a protocol recently reported elsewhere. ${ }^{25}$

\section{MATERIALS AND METHODS}

\section{Materials}

Unless otherwise stated, all materials were reagent grade. $\mathrm{PdCl}_{2}$ (99.9\%) was obtained from Wako Pure Chemical Industries Ltd., Osaka, Japan. Poly(4lithium styrene sulfonic acid) (PLSSA; nominal molecular weight $=75000$; $30 \mathrm{wt} \%$ aqueous solution), sodium chloride $(\mathrm{NaCl}, 99.5 \%)$, hydrated ferric chloride $\left(\mathrm{FeCl}_{3} \cdot 6 \mathrm{H}_{2} \mathrm{O}\right)$ and aluminum oxide (activated, basic, Brockmann 1, standard grade, $\sim 150$ mesh, $58 \AA$ ) were obtained from Sigma-Aldrich, St Louis, MO, USA and were used without further purification. Pyrrole (Py, 98\%) was also obtained from Sigma-Aldrich and was purified by passing through a column of activated basic aluminum oxide. Deionized water $\left(<0.06 \mu \mathrm{S} \mathrm{cm}^{-1}\right)$ was prepared using a deionized water producing apparatus (Advantec, MFS RFD240NA: GA25A-0715, Suite A Dublin, CA, USA) and was used for the synthesis and purification of the nanocomposite particles.

\section{Synthesis of PPy-Pd nanocomposite particles}

Chemical oxidative dispersion polymerizations were conducted as described previously. ${ }^{8}$ Briefly, Py $\left(20 \mathrm{mg}, 2.98 \times 10^{-4} \mathrm{~mol}\right)$ was added by syringe to an aqueous solution of PLSSA ( $400 \mathrm{wt} \%$ based on Py monomer, $1.0 \mathrm{ml}$ ) in a $13-\mathrm{ml}$ screw-capped bottle, and the system was stirred with a magnetic stir bar for $3 \mathrm{~h}$. $\mathrm{PdCl}_{2}$ oxidant $\left(62 \mathrm{mg}, 3.50 \times 10^{-4} \mathrm{~mol}\right)$ and $\mathrm{NaCl}(41 \mathrm{mg}$, $7.02 \times 10^{-4} \mathrm{~mol}$ ) were dissolved in $3.0 \mathrm{~g}$ water and then added to the aqueous solution of $\mathrm{Py}$ and colloidal stabilizer. $\mathrm{NaCl}$ (two molar equivalents to $\mathrm{PdCl}_{2}$ ) was added to dissolve the $\mathrm{PdCl}_{2}$ in the aqueous medium. The polymerizations were allowed to proceed for $24 \mathrm{~h}$ at $23^{\circ} \mathrm{C}$ and 200 r.p.m. The PPy-Pd nanocomposite particles were subsequently purified by repeated centrifugation-redispersion cycles (successive supernatants were replaced with deionized water) to remove the unwanted water-soluble chemicals (free colloidal stabilizer, $\mathrm{NaCl}$ and $\mathrm{HCl}$ ). Redispersion was achieved by sonication for more than $30 \mathrm{~min}$ using a Bransonic C221 (Yamato Scientific Co., Tokyo, Japan). PPy-Pd nanocomposite bulk powder was synthesized under the same conditions as the chemical oxidative dispersion polymerization described above, but without the use of colloidal stabilizer. PPy homopolymer bulk powder was synthesized by chemical oxidative precipitation polymerization using $\mathrm{FeCl}_{3}$ as an oxidant. The samples were washed more than 10 times with deionized water and then freeze dried overnight.

\section{Characterization of PPy-Pd nanocomposite particles}

TEM study. A droplet of aqueous dispersion of the PPy-Pd nanocomposite particles was placed on a TEM grid and dried to fix the particles on the plate. The structures and morphology of the collected nanocomposite particles were observed by a high-resolution TEM (JEM-2100F, JEOL, Tokyo, Japan). Using high-resolution TEM, it is possible to characterize the crystalline structure of the Pd component; this level of characterization was not possible by general TEM in our previous study. ${ }^{8}$ The number-average equivalent circle diameter (Heywood diameter, $D_{\mathrm{H}}$ ) was determined by counting more than 150 particles in several TEM images. The degree of circularity was evaluated using the following equation:

$$
\text { Circularity }=4 \pi S / l^{2},
$$

where $S$ is an equivalent circle area and $l$ is the circumference of the nanocomposite particle. A $S$ value close to 1 indicates a morphology close to a sphere.

\section{Dynamic light scattering}

Dynamic light scattering measurements were carried out on a Malvern Zeta Sizer Nano Series Nano-ZS instrument using a $4.0-\mathrm{mW}$ He-Ne laser $(\lambda=633$ $\mathrm{nm})$ at $25^{\circ} \mathrm{C}$.

\section{SAXS}

SAXS measurements were conducted on an aqueous dispersion of nanocomposite particles using a SAXS beamline (BL-10C) at the Photon Factory in the Research Organization for High Energy Accelerator (Tsukuba, Japan). The wavelength of the incident X-ray, $\lambda$, was tuned to $0.1488 \mathrm{~nm}$. The camera length (that is, distance from the sample position to the detector) was set to $2036 \mathrm{~mm}$. Additional details on the SAXS apparatus are available in the literature. ${ }^{26}$ An aqueous dispersion of nanocomposite particles was contained in a 1-mm-thick sample holder sealed with a thin polyimide film (TORAYDuPont Kapton film, Du Pont - Toray Co., Ltd., Tokyo, Japan; $25 \mu \mathrm{m}$ thick) and was subjected to SAXS analysis at room temperature $\left(c a 25^{\circ} \mathrm{C}\right)$ with a measuring time of $10 \mathrm{~s}$. The two-dimensional-SAXS patterns were recorded on a R-AXIS VII (Rigaku Co., Ltd., Tokyo, Japan; two-dimensional detector) and were converted to a one-dimensional (1d-SAXS) profile by conducting the circular average. Furthermore, the scattering of pure water was measured for $100 \mathrm{~s}$ and was subtracted from each of the 1d-SAXS profiles for the aqueous dispersion samples (background subtraction), after being normalized to the measuring time and absorption of the X-ray beam by the aqueous dispersion sample or pure water (transmission correction).

\section{Chemical composition}

The Pd and PPy loadings of the nanocomposite particles were determined by comparing the nitrogen content of the PPy-Pd nanocomposite particles with that of the PPy-Pd and PPy bulk powders. The nitrogen contents were determined by CHN elemental microanalysis (Yanaco CHN, Kyoto, Japan, Corder MT-5)

\section{RESULTS AND DISCUSSION}

The PPy-Pd nanocomposite particles were obtained as a black-colored aqueous dispersion, which was colloidally stable over 7 months without coagulation, by chemical oxidative dispersion polymerization in the presence of PLSSA. In this process, PLSSA was adsorbed on the particle surface and served as a colloidal stabilizer for the PPy-Pd nanocomposite particles. By contrast, the PPy-Pd nanocomposite was obtained as a floc by chemical oxidative precipitation polymerization in the absence of PLSSA. The hydrodynamic diameter of the nanocomposite particles was determined to be $89 \mathrm{~nm}$ by dynamic light scattering. Elemental microanalytical studies indicated the 
PPy-Pd nanocomposite bulk powder consisted of $40.1 \mathrm{wt} \%$ PPy and $59.9 \mathrm{wt} \% \mathrm{Pd}$ components, which is in good agreement with the theoretical values (38.3 wt\% PPy and $61.7 \mathrm{wt} \% \mathrm{Pd}$ ) as well as values measured in our previous studies. ${ }^{8,14,15}$ This result indicates that the Py was quantitatively polymerized with the $\mathrm{Pd}^{2}+$ oxidant. The weight ratio of the PPy and Pd components in the PPy-Pd nanocomposite particles was calculated to be 40.1:59.9, which is again in good agreement with the theoretical value. These results indicate that PLSSA does not interfere with the chemical oxidative polymerization of Py using $\mathrm{Pd}^{2+}$. PLSSA loading was determined to be $52.1 \mathrm{wt} \%$, suggesting that PLSSA existed within the particles as well as on their surfaces. ${ }^{8}$ The sulfonate group is known to be an efficient dopant anion for PPy and has stronger interactions with PPy compared with chloride ions. ${ }^{8,27,28}$ It has been confirmed that the PPy-Pd nanocomposite particles were doped with sulfonate groups rather than chloride ions by X-ray photoelectron spectroscopy studies. ${ }^{8}$ Therefore, it can be expected that the PLSSA colloidal stabilizer is adsorbed onto the surfaces of the PPy-Pd nanocomposite particles as a polymeric dopant anion (Figure 1).

The nanomorphology of the PPy-Pd nanocomposite particles was investigated by high-resolution TEM in the dry state (Figure 2). The TEM images show the presence of discrete, nanometer-sized non-spherical particles on the TEM grid. The $D_{\mathrm{H}}$ and circularity values were measured to be $32.9 \pm 21.6 \mathrm{~nm}$ and 0.51 , respectively. Polymer particles formed by dispersion polymerization are generally spherical because it is thermodynamically stable to minimize the area of the polymer-media interface. Thus, the non-spherical nanocomposite particles synthesized in this study seem to be kinetically locked rather than thermodynamically stable. PPy cannot be swollen with Py monomer and water and it is expected that non-swollen 'hard' primary nuclei consisting of PPy-Pd nanocomposites coagulated to produce a non-spherical morphology. The $D_{\mathrm{H}}$ was smaller than the hydrodynamic diameter, and this difference was attributed to the presence of the PLSSA colloidal stabilizer layer on the surface of the PPy-Pd nanocomposite particles. The PLSSA protective layer was hydrated and swollen in aqueous medium and then dehydrated and shrunken in the dry state. The heterogeneous character of the nanocomposite particles was ascribed to a more transparent host material (presumably the PPy and PLSSA) containing crystalline nanoparticles, which were identified as $\mathrm{Pd}$ (Figures $2 \mathrm{~b}$ and $\mathrm{c}$ ). The $D_{\mathrm{H}}$ and circularity values for the Pd nanoparticles were measured to be $5.4 \pm 0.99 \mathrm{~nm}$ and 0.61 , respectively.
Notably, high-resolution TEM studies suffer from a deficiency in analyzing three-dimensional morphology because three-dimensional structures are reduced to a two-dimensional image. Therefore, TEM images show overlapping Pd nanoparticles within the nanocomposite particles. Because SAXS is the appropriate method for evaluating spatial morphology on the nanometer scale, it was applied to characterize the spatial nanomorphology of the PPy-Pd nanocomposite particles. Because of its low electron density, which is close to that of water, the hydrated PLSSA colloidal stabilizer layer should be negligible in the SAXS studies compared with the PPy-Pd nanocomposite core component.

The 1d-SAXS profiles of 1, 2 and 3\% aqueous dispersions of the nanocomposite particles are shown together in Figure $3 \mathrm{a}$ as a plot of $\log [\mathrm{I}(q)]$ vs $\log q$, where $q$ is the magnitude of the scattering vector given by $q=(4 \pi / \lambda) \sin (\theta / 2)$, with $\lambda$ and $\theta$ being the $\mathrm{X}$-ray wavelength and the scattering angle, respectively. This plot clearly shows that the shapes of the profiles are similar. When the curves are vertically shifted, all of the data collapse onto a single curve (see Figure 3b), suggesting that the nanocomposite particles are dispersed in the aqueous medium without ordering into a lattice, at least up to a particle concentration of $3 \%$. Thus, the 1d-SAXS profile can be attributed directly to particle scattering. Although the TEM results revealed that the nanocomposite particles were not spherical, a mathematical equation describing particle scattering is not available for such an unusual shape of particles. Therefore, a spherical shape was assumed for simplicity. The theoretical particle scattering intensity, $I(q)$, for particles with a distribution of sizes can then be given as

$$
\begin{aligned}
& \mathrm{I}(q)=k \sum\left\{n(R)\left(4 \mathrm{p} R^{3} / 3\right)^{2}[\Phi(q)]^{2}\right\} \\
& \text { with } \Phi(q)=3 /(q R)^{3}[\sin (q R)-q R \cos (q R)]
\end{aligned}
$$

where $k$ is a numerical constant and $n(R)$ is the number fraction of spheres with a radius of $R$, providing the size distribution of spheres. Attempts to fit a theoretical function given by equation (1) to the measured 1d-SAXS profile assuming a Gaussian or Schulz-Zimm type distribution for $n(R)$ were unsuccessful. We then employed a protocol in which $n(R)$ was directly determined by fitting the calculated $I(q)$ from equation (1) to the experimentally observed 1d-SAXS profile. ${ }^{25}$ The least-squares fitting was conducted by floating the following parameters as the fitting parameters: the numerical constant $k$ and the abundance number of particles having the size $R$ in the step of $1 \mathrm{~nm}$ (that is, $n(R=1 \mathrm{~nm}), n(R=2 \mathrm{~nm}), n(R=3 \mathrm{~nm}), \ldots n(R=40 \mathrm{~nm})$ ).

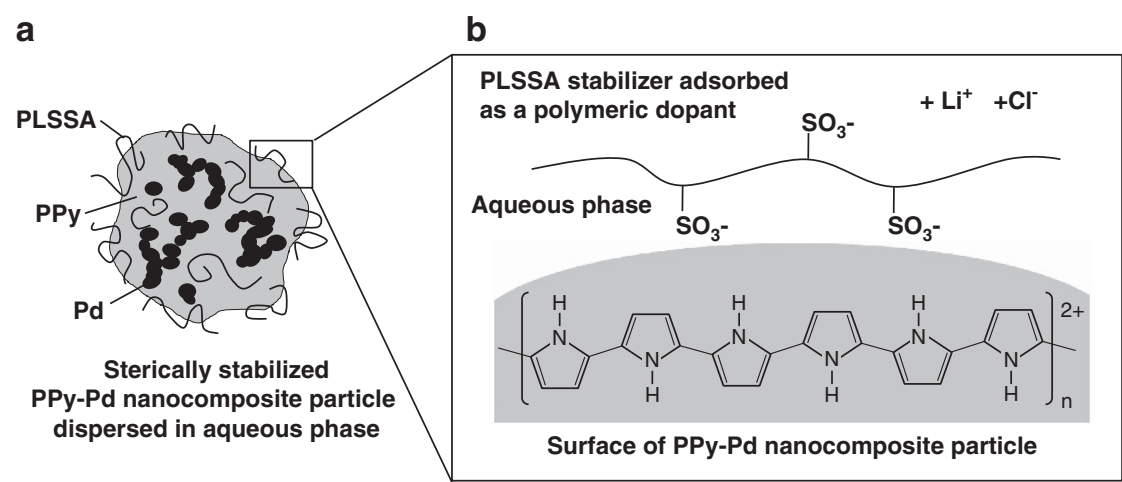

Figure 1 (a) Sterically stabilized PPy-Pd nanocomposite particles synthesized by the chemical oxidative dispersion polymerization of pyrrole using palladium (II) chloride in the presence of poly(4-lithium styrene sulfonic acid) (PLSSA) as a colloidal stabilizer. (b) Schematic representation of the surface interaction between the PLSSA stabilizer and the PPy component of the PPy-Pd nanocomposite particles. The polyelectrolyte stabilizer acts as a polymeric dopant anion for the cationic PPy chains, leading to the concomitant expulsion of $\mathrm{Li}^{+}$and $\mathrm{Cl}^{-}$counter anions. 


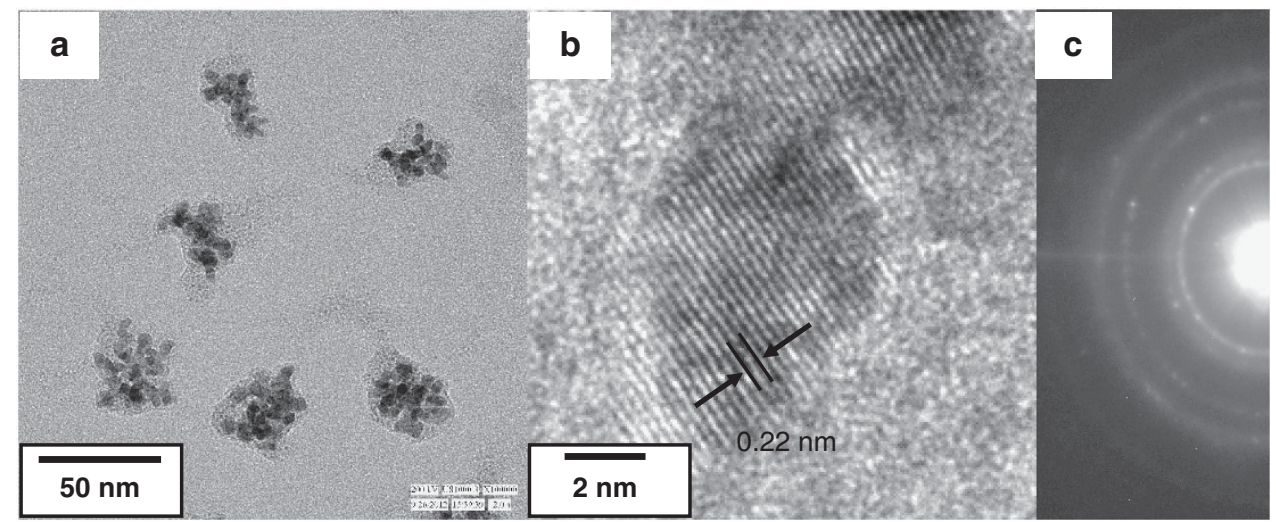

Figure 2 TEM images of sterically stabilized PPy-Pd nanocomposite particles synthesized by aqueous chemical oxidative dispersion polymerization. (a) Lowmagnification image of the particles. (b) High-resolution TEM image of a Pd nanocrystal observed in a. (c) selected area electron diffraction pattern corresponding to the image in a.
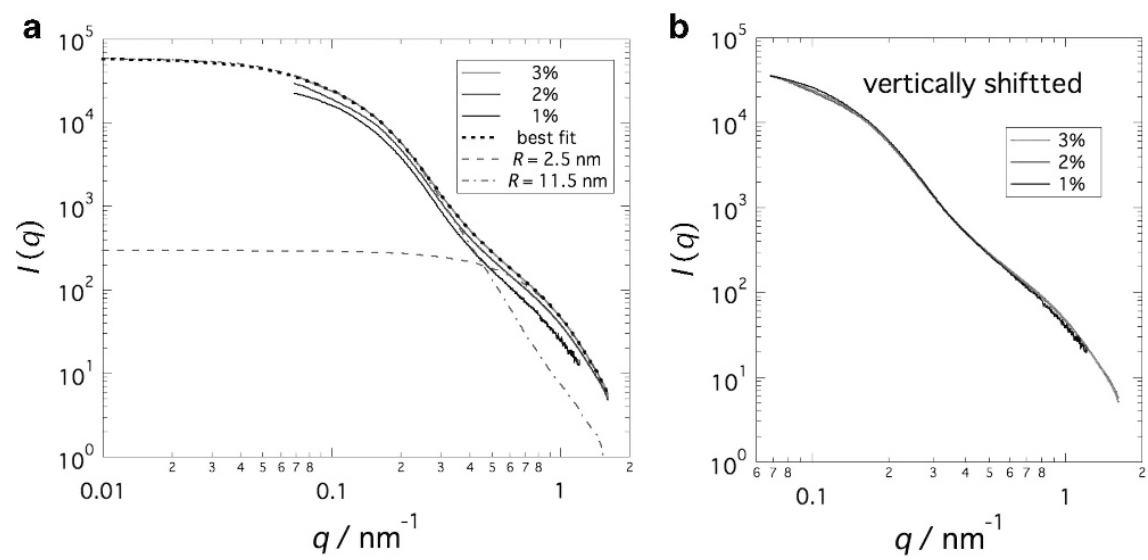

Figure 3 (a) One-dimensional SAXS profiles for 1, 2 and 3\% aqueous dispersions of the nanocomposite particles as shown in a plot of log [I( $q)$ ] vs log $q$, where $q$ is the magnitude of the scattering vector. The best-fit curve obtained is shown as a dotted black curve on the $1 \mathrm{~d}$-SAXS profile for the $3 \%$ aqueous solution (the most intense one and, therefore, the most reliable). SAXS curves calculated by assuming $n(R)$ with a single peak at approximately $R=2.5 \mathrm{~nm}$ and approximately $R=11.5 \mathrm{~nm}$ are shown as a broken curve and a dotted-and-broken curve, respectively. (b) Master curve for the 1d-SAXS profile obtained vertically shifting the three 1d-SAXS profiles shown in Figure $3 a$ (the curves are not shifted in the $q$-axis direction). A full color version of this figure is available at Polymer Journal online.

This protocol worked well, and the best fit obtained is shown by a dotted black curve on the 1d-SAXS profile for the $3 \%$ aqueous solution (the most intense one and therefore the most reliable) in Figure 3a. The particle size distribution is shown in Figure 4. The size distribution is shown in the units of vol\% and was calculated using $n(R)$ and the following equation:

$$
\text { abundance }(\operatorname{vol} \%)=n(R) R^{3} / \sum\left\{n(R) R^{3}\right\} \times 100
$$

The reason why we employed vol\% instead of $n(R)$ is that the numbers of larger particles close to $R=11.5 \mathrm{~nm}$ were too small compared with those around $R=2.5 \mathrm{~nm}$, making them invisible on the plot of $n(R)$. Furthermore, expressing abundance in the unit of vol\% is more suitable and corresponds well with the results of visual observation, where larger particles can be easily identified, even if they are only present in small numbers.

From the results, it is clear that the size distribution of the particles was bimodal, not unimodal, and two peaks could be identified at approximately $R=2.5$ and $11.5 \mathrm{~nm}$. To ensure that the results of the size distribution $n(R)$ were reliable, the single contributions from each of the peaks in $n(R)$ to the SAXS profile are examined in Figure 3a. Importantly, SAXS curves calculated by assuming $n(R)$ with a single

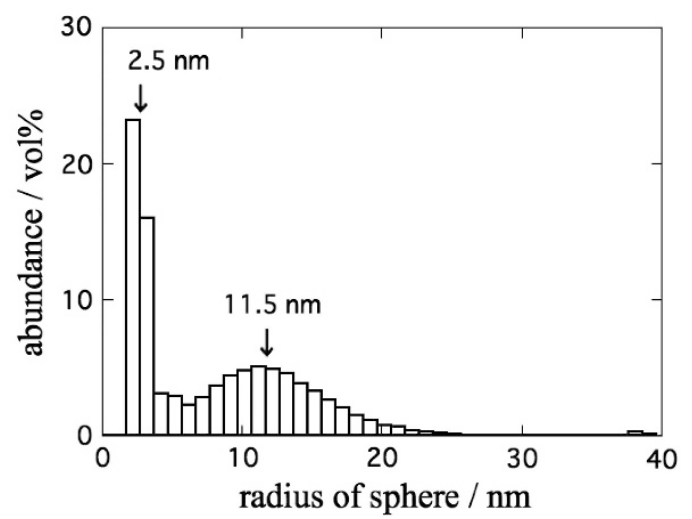

Figure 4 Distribution of the radius of spherical nanoparticles, as determined by fitting the particle scattering data (the SAXS profile).

peak either at approximately $R=2.5 \mathrm{~nm}$ or approximately $R=11.5$ $\mathrm{nm}$ could perfectly explain the measured SAXS profile. It is recognized, however, that the smaller particles could explain the 
shape of the SAXS profile in the higher $q$ range (see the broken curve), whereas the larger ones characterized the SAXS profile in the lower $q$ range (see the dotted-and-broken curve). Here, a simple question arises as to whether this result indicates the real distribution of the nanocomposite particle itself. The answer is no because the TEM observations (Figure 2) suggest that the average radius was approximately $16 \mathrm{~nm}$ with a unimodal distribution. (Note that SAXS studies measure the radius of the PPy-Pd nanocomposite core without the hydrated PLSSA colloidal stabilizer layer, and the diameters determined by TEM and SAXS technique were close to each other.) Thus, these results imply that the additional unimodal distribution with the peak at $R=2.5 \mathrm{~nm}$ may be attributed to the size distribution of $\mathrm{Pd}$ nanoparticles existing in the nanocomposite particles. This speculation can be confirmed by close examination of the high-resolution TEM image; the Pd nanoparticle size estimated by SAXS studies agreed well with that determined by TEM studies ( $R=2.7 \mathrm{~nm}$; Figure 2). It should be noted that the Pd nanoparticles were randomly distributed in the nanocomposite particles, as shown in Figure 2. Therefore, a straightforward core-shell model is not ideal for calculating the particle scattering for the SAXS profile fitting. Nevertheless, even in this unfavorable situation, it was found that the SAXS results could be accounted for by a simple summation of the particle scattering. Furthermore, the wide distribution of the $\mathrm{Pd}$ nanoparticle diameters may imply aggregation of the Pd nanoparticles. Indeed, some TEM images show overlapping of the $\mathrm{Pd}$ nanoparticles, which could be simply considered a result of aggregation, rather than the superimposition of discrete particles in the twodimensional images (as stated above).

In addition, it should be noted that the size distribution of the PPyPd nanocomposite particles was much wider compared with that of the Pd nanoparticles. This difference could indicate that the distance between the Pd nanoparticles was not uniformly controlled, and/or the number of the Pd nanoparticles per nanocomposite particle was relatively widely distributed, whereas the $\mathrm{Pd}$ nanoparticles were relatively uniform.

\section{CONCLUSIONS}

In summary, high-resolution TEM studies revealed that PPy-Pd nanocomposite particles had non-spherical shapes with a heterogeneous nanomorphology composed of a PPy matrix containing $\sim 5 \mathrm{~nm}$ Pd nanoparticles. SAXS studies succeeded in measuring the diameters of the PPy-Pd nanocomposite particles as well as the Pd nanocrystal particles in aqueous medium, and the average diameters for the nanocomposite particles and the Pd nanocrystal particles were 23.0 and $5.0 \mathrm{~nm}$, respectively. The SAXS results were in good agreement with the TEM results. The particle size distributions were also characterized by using SAXS to measure the radius of the PPy-Pd nanocomposite core without the hydrated PLSSA colloidal stabilizer layer. The diameters determined by TEM and SAXS were similar but were smaller than the hydrodynamic diameter determined by dynamic light scattering. The synthesized nanocomposite particles are expected to function as a catalyst for organic reactions (for example, the Suzuki-Miyaura coupling reaction) performed in aqueous media. ${ }^{14,15}$

\section{ACKNOWLEDGEMENTS}

Professor Hiroya Abe and Eri Nakamura (Joining and Welding Research Institute, Osaka University) are thanked for their assistance with the TEM studies. Professor Shin-ichi Yusa (Hyogo U.) is thanked for his assistance with the dynamic light scattering study. This work was partially supported by a Grant-in-Aid for Scientific Research on Innovative Areas 'New Polymeric
Materials Based on Element-Blocks (25102542)' and 'Engineering NeoBiomimetics (25120511)' from the Ministry of Education, Culture, Sports, Science and Technology of Japan and by the Science Research Promotion Fund from the Promotion and Mutual Aid Corporation for Private Schools of Japan (No. 51). The SAXS measurements were performed at KEK-PF (BL-10C) with the approval number $2011 \mathrm{G} 612$.

1 Gangopadhyay, R. \& De, A. Conducting polymer nanocomposites: a brief overview. Chem. Mater. 12, 608-622 (2000).

2 Fujii, S. \& Nakamura, Y. in: Applied Surface Science of Nanomaterials. (eds Pinson, J. \& Chehimi, M. M.) Ch 11, 303-317 (Nova Science Publishers, New York, USA, 2013).

3 Marinakos, S. M., Novak, J. P., Brousseau, L. C., House, B. A., Edeki, E. M., Feldhaus, J. C. \& Feldheim, D. L. Gold particles as templates for the synthesis of hollow polymer capsules. control of capsule dimensions and guest encapsulation. J. Am. Chem. Soc. 121, 8518-8522 (1999).

4 Attia, M. F., Azib, T., Salmi, Z., Singh, A., Decorse, P., Battaglini, N., Lecoq, H., Omastová, M., Higazy, A. A., Elshafei, A. M., Hashem, M. M. \& Chehimi, M. M. Onestep UV-induced modification of cellulose fabrics by polypyrrole/silver nanocomposite films. J.Colloid Interface Sci. 393, 130-137 (2013).

5 Fujii, S., Aichi, A., Akamatsu, K., Nawafune, H. \& Nakamura, Y. One-step synthesis of polypyrrole-coated silver nanocomposite particles and their application as a coloured particulate emulsifier. J. Mater. Chem. 17, 3777-3779 (2007),

6 Freund, M. S., Henry, M. C., Hsueh, C. C. \& Timko, B. P. Reaction of pyrrole and chlorauric acid: a new route to composite colloids. J. Electrochem. Soc. 148, 155-162 (2001).

7 Vasilyeva, S. V., Vorotyntsev, M. A., Bezverkhyy, I., Lesniewska, E., Heintz, O. \& Chassagnon, R. Synthesis and characterization of palladium nanoparticle/polypyrrole composites. J. Phys. Chem. C 112, 19878-19885 (2008).

8 Hamasaki, H., Fukui, N., Fujii, S., Yusa, S. \& Nakamura, Y. Sterically stabilized polypyrrole-palladium nanocomposite particles synthesized by aqueous chemical oxidative dispersion polymerization. Colloid Polym. Sci. 291, 223-230 (2013).

9 Zhao, M. \& Crooks, R. M. Homogeneous hydrogenation catalysis with monodisperse, dendrimer-encapsulated Pd and Pt nanoparticles. Angew. Chem. Int. Ed. 38, 364-366 (1999).

10 Crooks, R. M., Zhao, M., Sun, L., Chechik, V. \& Yeung, L. K. Dendrimer-encapsulated metal nanoparticles: synthesis, characterization, and applications to catalysis. Acc. Chem. Res. 34, 181-190 (2001).

11 Reetz, M. T. \& Westermann, E. Phosphane-free palladium-catalyzed coupling reactions: the decisive role of Pd nanoparticles. Angew. Chem. Int. Ed. 39, 165-168 (2000).

12 Reetz, M. T., Lohmer, G. \& Schwickardi, R. A new catalyst system for the heck reaction of unreactive aryl halides. Angew. Chem. Int. Ed. 37, 481-483 (1998).

13 Klingelhöfer, S., Heitz, W., Greiner, A., Oestreich, S., Förster, S. \& Antonietti, M. Preparation of palladium colloids in block copolymer micelles and their use for the catalysis of the heck reaction. J. Am. Chem. Soc. 119, 10116-10120 (1997).

14 Fujii, S., Matsuzawa, S., Nakamura, Y., Ohtaka, A., Teratani, T., Akamatsu, K., Tsuruoka, T. \& Nawafune, H. Synthesis and characterization of polypyrrole-palladium nanocomposite-coated latex particles and their use as a catalyst for Suzuki coupling reaction in aqueous media. Langmuir 26, 6230-6239 (2010).

15 Fujii, S., Hamasaki, H., Abe, H., Yamanaka, S., Ohtaka, A., Nakamura, E. \& Nakamura, Y. One-step synthesis of magnetic iron-conducting polymer-palladium ternary nanocomposite microspheres and their use as recyclable catalyst. J. Mater Chem. A 1, 4227-4430 (2013).

16 Pedersen, J. S. Analysis of small-angle scattering data from colloids and polymer solutions: modeling and least-squares fitting. Adv. Colloid Interface Sci. 70, 171-210 (1997).

17 Abécassis, B., Testard, F., Spalla, O. \& Barboux, P. Probing in situ the nucleation and growth of gold nanoparticles by small-angle X-ray scattering. Nano Lett. 7, 1723-1727 (2007).

18 Polte, J., Emmerling, F., Radtke, M., Reinholz, U., Riesemeier, H. \& Thünemann, A. F. Real-time monitoring of copolymer stabilized growing gold nanoparticles. Langmuir 26, 5889-5894 (2010)

19 Polte, J., Erler, R., Thünemann, A. F., Sokolov, S., Ahner, T. T., Rademann, K., Emmerling, F. \& Kraehnert, R. Nucleation and growth of gold nanoparticles studied via in situ small angle X-ray scattering at millisecond time resolution. ACS Nano 4, 1076-1082 (2010).

20 Harada, M. \& Katagiri, E. Mechanism of silver particle formation during photoreduction using in situ time-resolved SAXS analysis. Langmuir 26, 17896-17905 (2010).

21 Harada, M., Tamura, N. \& Takenaka, M. Nucleation and growth of metal nanoparticles during photoreduction using in situ time-resolved SAXS analysis. J. Phys. Chem. C 115, 14081-14092 (2011).

22 Tanaka, H., Koizumi, S., Hashimoto, T., Itoh, H., Satoh, M., Naka, K. \& Chujo, Y. Combined in situ and time-resolved SANS and SAXS studies of chemical reactions at specific sites and self-assembling processes of reaction products: reduction of palladium ions in self-assembled polyamidoamine dendrimers as a template. Macromolecules 40, 4327-4337 (2007).

23 Balmer, J. A., Mykhaylyk, O. O., Armes, S. P., Fairclough, J. P., Ryan, A. J., Gummel, J., Murray, M. W., Murray, K. A. \& Williams, N. S. Time-resolved small-angle 
X-ray scattering studies of polymer-silica nanocomposite particles: initial formation and subsequent silica redistribution. J. Am. Chem. Soc. 133, 826-837 (2011).

24 Balmer, J. A., Mykhaylyk, O. O., Schmid, A., Armes, S. P., Fairclough, J. P. \& Ryan, A. J. Characterization of polymer-silica nanocomposite particles with core-shell morphologies using Monte Carlo simulations and small angle X-ray scattering. Langmuir 27, 8075-8089 (2011).

25 Tien, N. D., Hoa, T. P., Mochizuki, M., Saijo, K., Hasegawa, H., Sasaki, S. \& Sakurai, S. Higher-order crystalline structures of poly(oxyethylene) in poly(D, L-lactide)/poly(oxyethylene) blends. Polymer 54, 4653-4659 (2013)
26 Ueki, T., Hiiragi, Y., Kataoka, M., Inoko, Y., Amemiya, Y., Izumi, Y., Tagawa, H. \& Muroga, Y. Aggregation of bovine serum albumin upon cleavage of its disulfide bonds, studied by the time-resolved small-angle $\mathrm{X}$-ray scattering technique with synchrotron radiation. Biophys. Chem. 23, 115-124 (1985).

27 Münstedt, H. Properties of polypyrroles treated with base and acid. Polymer 27, 899-904 (1986).

28 Beadle, P. M., Armes, S. P., Greaves, S. J. \& Watts, J. F. X-ray photoelectronspectroscopy studies on sterically-stabilized polypyrrole particles. Langmuir 12, 1784-1788 (1996). 